\title{
Water alteration on (42355) Typhon?
}

\author{
Alvaro Alvarez-Candal ${ }^{1}$ and Maria Antonietta Barucci ${ }^{2}$ \\ ${ }^{1}$ European Souther Observatory, \\ Alonso de Córdova 3107, Vitacura Casilla 19001, Santiago 19, Chile \\ email: aalvarez@eso.org \\ ${ }^{2}$ LESIA/Observatoire de Paris \\ 5, Place Jules Janssen, 92195, Meudon, France
}

\begin{abstract}
The visible spectra of (42355) Typhon showed evidence for aqueously altered materials. Therefore we seek to understand if such an event is possible.

We use data from the ESO / Very Large Telescope together with the Hapke Hapke radiative transfer model to interpret the surface composition of (42355) Typhon over the whole spectral range $(\sim 0.5-2.4 \mu \mathrm{m})$.

Our results points that (42355) Typhon could be a fragment from a larger parent body that suffered aqueous alteration.
\end{abstract}

Keywords. techniques: spectroscopic, Kuiper Belt

\section{Introduction}

Aiming at obtaining high quality data of the trans-Neptunian objects, TNOs, population, a large program for the observation of centaurs and TNOs was started using the facilities of the ESO / Very Large Telescope at Cerro Paranal in Chile (PI: M.A. Barucci). The program lasted from November 2006 until December 2008. In the framework of the large program we observed the scattered disk object, SDO, (42355) Typhon.

Its spectrum shows evidence of water ice in the near infrared (Guilbert et al. 2009a). Although no signature of ices are found in the visible, in a previous work we detected a subtle absorption feature possibly related to aqueously altered material (Alvarez-Candal et al. 2008, AC08 hereafter). (42355) Typhon is also the primary body of a binary system (Noll et al. 2006). Its diameter is about $175 \mathrm{~km}$ (Stansberry et al. 2008), however, this measurement could be overestimated due to its binary nature. Note that recently Benecchi et al. (2009) showed that the colors of both bodies in the system are similar.

\section{Analysis}

The data we analize here was obtained during two runs at the Very Large Telescope in January 2007 and April 2008, using FORS2 and ISAAC at the UT 1 and SINFONI at the UT 4. We use also data already published: VRIJHK photometry (DeMeo et al. 2009), visible spectroscopy by AC08, and H+K spectroscopy by Guilbert et al. (2009a). Therefore, in what follows, we concentrate on the results obtained from the modeling and our interpretation of the data.

Figure 1 shows the two composite spectra of (42355) Typhon, from 0.4 to $2.3 \mu \mathrm{m}$. To connect the entire spectral range we used the colors (V-J) and (V-H) from DeMeo et al. (2009). The spectral gradient, $S^{\prime}$, in the visible of both spectra are similar, 10.6 \pm 0.6 and $11.7 \pm 0.5 \%(0.1 \mu \mathrm{m})^{-1}$, for the January 2007 and April 2008 observations, respectively.

As we suggested in AC08 that (42355) Typhon shows evidence of water alterarion, we processed both visible spectra adapting the technique described in Vilas et al. 


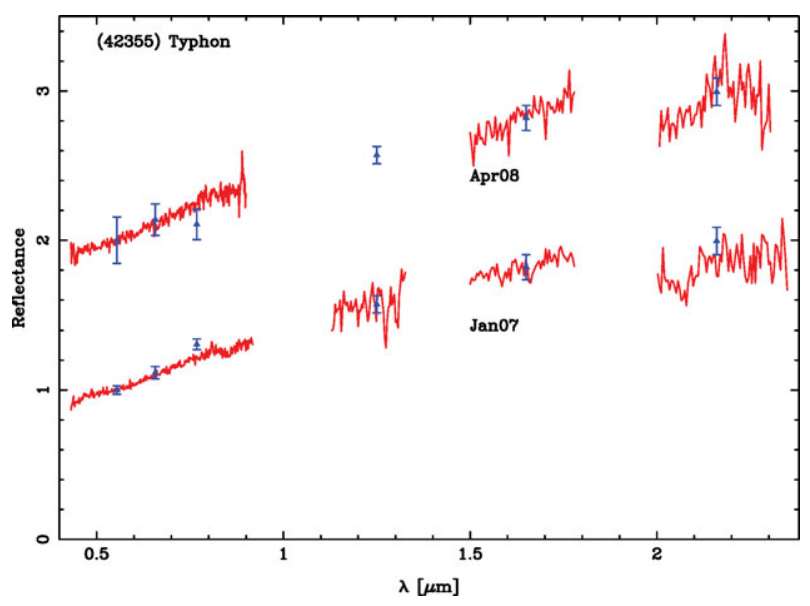

Figure 1. Composite spectrum of (42355) Typhon obtained with the data taken in the January 2007 and April 2008 runs. The spectra are normalized to unity at the V-filter, April 2008 spectrum is shifted by 1 for clarity. The triangles show the photometric colors used to set the reflectance scale. The region between 1.8 and $2.0 \mu \mathrm{m}$ was removed due to the strong telluric absorptions.

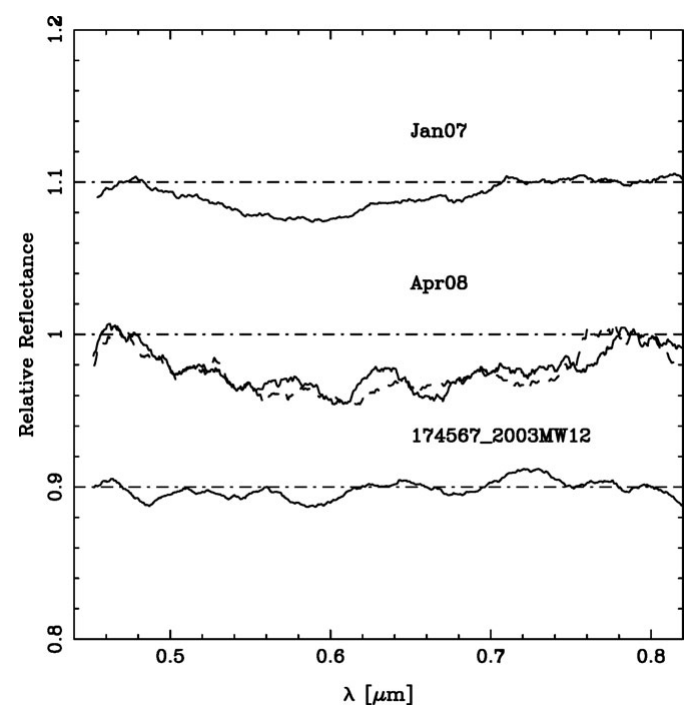

Figure 2. Visible spectra of (42355) Typhon after extraction of the continuum. The spectra are offset by a reflectance of 0.1 for comparison. The dashed line indicate the spectrum of (42355) Typhon as obtained dividing by a different solar analog observed the same night of the April 2008 run. To illustrate that the absorption we see is not due to our choice of calibration star, we apply the same analysis to spectra of (174567) $2003 \mathrm{MW}_{12}$, obtained the same night as our (43355) Typhon data, and find no evidence for an absorption.

(1993, 1994): We smoothed the spectra using a window of about $0.03 \mu \mathrm{m}$, then we estimated the slope of the continuum, tracing a line between 0.47 and $0.78 \mu \mathrm{m}$ (e.g., Carvano et al. 2003). The spectra were then divided by this conventional continuum. These "normalized" spectra are shown in Fig. 2. There is evidence for the existence of an absorption feature in the April 2008 spectrum, even deeper than that observed in January 2007 although the shape and position are slightly different. 


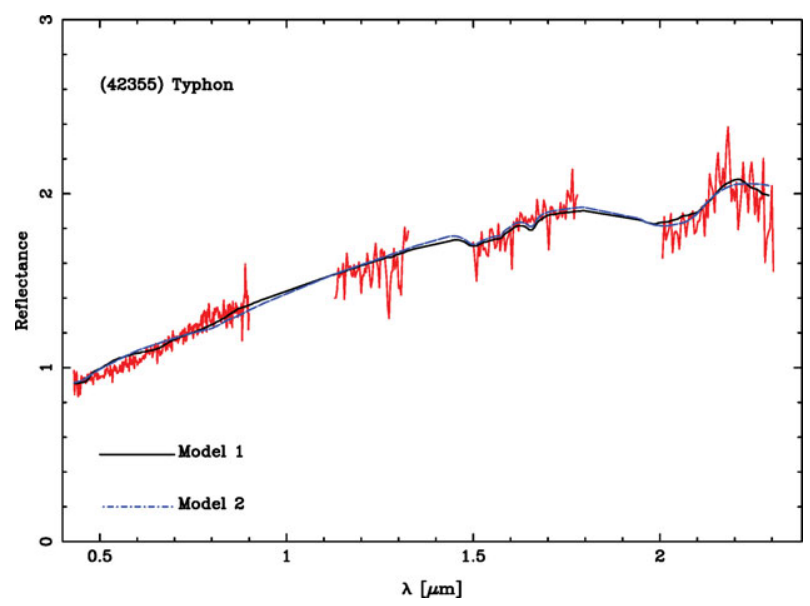

Figure 3. Composite spectra of (42355) Typhon taken in April 2008. The spectrum is normalized to unity at the V-filter. The best fitting models were obtained using intimate mixtures. The black line, Model 1, corresponds to a model including $12 \%$ Serpentine $(160 \mu \mathrm{m}), 4 \%$ of crystalline water ice $(85 \mu \mathrm{m}), 17$, and $6 \%$ of Triton and Titan tholins, respectively, and $61 \%$ of amorphous carbon. This model corresponds to an albedo of $6.38 \%$ at $0.55 \mu \mathrm{m}$. The dashed line, labeled Model 2, includes $6 \%$ of crystalline water ice $(45 \mu \mathrm{m}), 28 \%$ of Triton tholin $(13 \mu \mathrm{m})$, $59 \%$ of Titan tholin $(1 \mathrm{~mm}), 6 \%$ of amorphous water ice $(10 \mu \mathrm{m})$, and $1 \%$ of amorphous carbon. The albedo at $0.55 \mu \mathrm{m}$ is $6.04 \%$.

In the near infrared we only see a feature in the $\mathrm{H}+\mathrm{K}$ region which we associate with water ice. The apparent feature in the January 2007 spectrum at $2.25 \mu \mathrm{m}$ is probably an artifact of the combination of decreasing efficiency of the detector and the faintness of the target.

The April 2008 data was modeled using a radiative transfer model, based on the Hapke theory (Hapke, 1993). The free parameters of the model are the compound grain size and abundance. The best fit model was obtained by minimizing $\chi^{2}$ using a MarqvardtLevenberg algorithm.

We considered: Triton and Titan tholins, to match the red continuum in the visible (Khare et al. 1984, 1993), water ice in crystalline and amorphous states (crystalline water ice constants at $40 \mathrm{~K}$ from Quirico and Schmitt 1997, amorphous ice at $38 \mathrm{~K}$ from Grundy and Schmitt 1998), amorphous carbon to match the low albedo (Zubko et al. 1996). The only hydrated mineral for which optical constants are available is Serpentine (R. Clark private communication). Both models describe, reasonably well, the whole spectral range, although none of them is able to reproduce the feature detected in the visible.

\section{Discussion}

The feature observed at about $0.6 \mu \mathrm{m}$ resembles those found in some low albedo asteroids (Vilas et al., 1994, Rivkin et al., 2002), which are often associated to phyllosilicates, formed by the interaction of liquid water and silicates. It could indicate the presence of material that suffered aqueous alteration. Phyllosilicates could also have bands in the near infrared. The only ones covered by our observations are those between 2.2 and $2.4 \mu \mathrm{m}$. Unfortunately, our spectra do not have enough signal-to-noise ratio as to confidently confirm a detection of such features.

The decay of radiogenic material could produce enough heat as to melt the water ice in the interior of icy bodies (see McKinnon et al., 2008). This is supported by the detection 
of hydrated silicates on comet nuclei (Lisse et al., 2006). Nevertheless, (42355) Typhon is too small to have suffered this kind of process.

As (42355) Typhon is too small to have suffered alteration, it is still possible that it is a fragment of a larger parent body that did suffer it. SDOs are dynamically related to centaurs and JFCs, so further evidences could be find in these populations. Interestingly, Guilbert et al. (2009b) showed that the centaur (10199) Chariklo has a feature in the visible, probably indicating water alteration, similar to that of (42355) Typhon. (10199) Chariklo is twice as big as (42355) Typhon, therefore it is possible that it had enough internal heat to melt water ice, so as there exist a Chariklo, there could have existed a larger body that originated Typhon after a catastrophic collision.

\section{References}

Alvarez-Candal, A., Fornasier, S., Barucci, M. A., de Bergh, C., \& Merlin, F. 2008, A\&A, 487, 741

Benecchi, S. D., Noll, K. S., Grundy, W. M., Buie, W. M., Stephens, D. C., \& Levison, H. F. 2009, Icarus, 200, 292

Carvano, J. M., Mothé-Diniz, T., \& Lazzaro, D. 2003, Icarus, 161, 356

DeMeo, F., Fornasier, S., Barucci, M. A., Perna, D., Protopapa, S., Alvarez-Candal, A., Delsanti, A., Doressoundiram, A., Merlin, F., \& de Bergh, C. 2009, A $\& A$ A, 493, 283

Grundy, W. M. \& Schmitt, B. 1998, Journal Geophys. Res., 103, 25809

Guilbert, A., Alvarez-Candal, A., Merlin, F., Barucci, M. A., Dumas, C., de Bergh, C., \& Delsanti, A. 2009a, Icarus, 201, 272

Guilbert, A., Barucci, M. A., Brunetto, R., Delsanti, A., Merlin, F., Alvarez-Candal, A., Fornasier, S., de Bergh, C., \& Sarid, G. 2009b, A\& A, 501, 777

Hapke, B. 1993, Topics in Remote Sensing 3: Theory of reflectance and emittance spectroscopy, (Cambridge: Cambridge Univ. Press)

Khare, B. N., Sagan, C., Arakawa, E. T., Suits, F., Callcott, T. A., \& Williams, M. W. 1984, Icarus, 60,127

Khare, B. N., Thompson, W. R., Cheng, L., Chyba, C., Sagan, C., Arakawa, E. T., Meisse, C., \& Tuminello, P. S. 1993, Icarus, 103, 290

Lisse, C. M. \& 16 colleagues 2006, Nature, 313, 635

McKinnon, W. B., Prialnik, D., Stern, S. A., \& Coradini, A. 2008, in: M. A. Barucci, H. Boehnhardt, D., Cruikshank, \& A. Morbidelli (eds.), The Solar System Beyond Neptune, (Tucson: Univ. of Arizona Press), p. 213

Noll, K. S., Grundy, W. M., Stephens, D. C., \& Levison, H. F. 2006, IAU Circulars, 8689, 1

Quirico, E. \& Schmitt, B. 1997, Icarus, 127, 354

Rivkin, A. S., Howell, E. S., Vilas, F., \& Lebofsky, L. A. 2003, in: W. F. Bottke, A. Cellino, P. Paolicchi, \& R. P. Binzel (eds.), Asteroids III, (Tucson: Univ. of Arizona Press, Tucson), p. 235

Stansberry, J., Grundy, W., Brown, M., Cruikshank, D., Spencer, J., Trilling, D., \& Margot, J.-L. 2008, in: M. A. Barucci, H. Boehnhardt, D. Cruikshank, \& A. Morbidelli, (eds.), The Solar System Beyond Neptune, (Tucson: Univ. of Arizona Press), p. 161

Vilas, F., Hatch, E. C., Larson, S. M., Sawyer, S. R., \& Gaffey, M. J. 1993, Icarus, 102, 225

Vilas, F., Jarvis, K. S., \& Gaffey, M. J. 1994, Icarus, 109, 274

Zubko, V. G., Mennella, V., Colangeli, L., \& Bussoletti, E. 1996, MNRAS, 282, 1321 